\title{
Tacrolimus combined with low-dose corticosteroids is an effective and safe therapeutic option for refractory IgA nephropathy
}

\author{
QI-JUN WAN, HAO-FEI HU, YONG-CHENG HE, SHAO-DONG LUAN, \\ HONG-TAO CHEN, TONG LI, YI XU, HUI-LI XU and YING LIAO
}

\author{
Department of Nephrology, Shenzhen Second People's Hospital, Shenzhen, Guangdong 518035, P.R. China
}

Received May 7, 2015; Accepted June 14, 2016

DOI: $10.3892 /$ etm.2016.3523

\begin{abstract}
Tacrolimus (TAC) has been shown to improve remission from proteinuria in patients with refractory IgA nephropathy (IgAN); however, the efficacy and safety of TAC in such patients have not been fully explored. Therefore, the present study was conducted to evaluate the tolerance to and efficacy of TAC combined with low-dose corticosteroids in patients with refractory IgAN. This was a single-center retrospective study. A total of 28 patients with refractory IgAN were randomly included and received TAC plus corticosteroid; 26 patients received TAC and prednisone, and 2 patients received TAC and methylprednisolone. In addition, all patients were treated with an angiotensin inhibitor. Total urinary protein excretion, serum albumin, blood glucose, complete remission (CR), partial remission (PR), cholesterol, low-density lipoprotein (LDL), serum creatinine (Scr) and estimated GFR (eGFR) were tested at baseline and at 3,6 and 12 months after the initiation of treatment in all patients. The primary endpoints were CR and PR. Secondary endpoints included changes of Scr, eGFR, clinical data and adverse events. After 12 months, CR was achieved in $40.1 \%$ of patients and PR in $43.4 \%$, yielding a total response rate of $83.5 \%$, and the total urinary protein excretion, serum albumin, cholesterol and LDL results were improved significantly compared with those at baseline. Proteinuria and serum albumin results were significantly improved by month 3 of treatment. Two patients relapsed during months 3-6 of follow-up. At the 12-month follow-up, renal function was improved compared with the baseline level as evidenced by eGFR and Scr, respectively. The blood glucose level was stable. One case of pneumococcal pneumonia developed in a patient treated with TAC plus low-dose methylprednisolone and one case of upper gastrointestinal hemorrhage was found in a patient treated with TAC
\end{abstract}

Correspondence to: Dr Yong-Cheng He, Department of Nephrology, Shenzhen Second People's Hospital, 3002 Sungang West Road, Shenzhen, Guangdong 518035, P.R. China E-mail: heyccheng@126.com

Key words: IgA nephropathy, tacrolimus, prednisone, methylprednisolone, renal pathology, adverse events plus low-dose prednisone; both cases completely recovered after treatment. In conclusion, TAC combined with low-dose corticosteroids may be an effective and safe therapeutic option for the treatment of refractory IgAN. However, given the small number of patients in this study, further prospective randomized controlled trials are required with a larger sample of participants and longer follow-up period.

\section{Introduction}

Ig A nephropathy (IgAN) is an immune-complex-mediated glomerulonephritis, and is the most common form of primary glomerular disease worldwide (1). A substantial number of patients with IgAN succumb to end-stage renal disease, a condition that requires renal-replacement therapy (2). IgAN occurs in individuals at any age, predominantly among males, with Caucasian and Asian populations being more prone to IgAN than individuals of African descent in the United States and South Africa $(3,4)$. Renal prognostic factors have been found to include impaired renal function, proteinuria, hypertension and advanced histological lesions (5). Atherosclerotic metabolic factors, including fasting triglyceride levels, insulin resistance, obesity and uric acid have also been shown to be associated with the renal prognosis of $\operatorname{IgAN}(6,7)$.

Previous studies have shown that tacrolimus (TAC), a potent calcineurin inhibitor, is effective in the treatment of nephritic syndrome, particularly in refractory nephritic syndrome in children and adult patients $(8,9)$. TAC was first applied as an immunosuppressant in transplant patients, and has been successfully used in heart, liver, kidney and pancreas transplantation, with excellent short-term outcomes (10-12). For example, TAC treatment does not disturb lipid metabolism or the antioxidant status of the body; furthermore, it protects neuronal tissue from hypoxic insults $(13,14)$. The immunomodulatory action of TAC is dependent upon its ability to disrupt the activation of $\mathrm{T}$ cells and the expression of cytokines (15). Significantly, calcineurin inhibitors may have an anti-proteinuric action through an effect on the stability of the podocyte cytoskeleton $(16,17)$.

Corticosteroids are a critical component of immunosuppressive regimens, yet the prolonged use of corticosteroids may reduce defensive responses among patients in the high-relevance region and result in various side effects, including hyperlipidemia, hypertension and diabetes mellitus (18). 
Elimination of the negative effects of corticosteroids from immunosuppressive regimens was therefore necessary during the treatments administered in the present study.

Although TAC is able to provide improved remission from proteinuria in patients with refractory IgAN (19), the effectiveness of TAC combined with low-dose corticosteroids in Chinese patients with Lee class III, IV or V IgAN and the tolerance to the treatment are unclear. The present study was conducted to evaluate the tolerance to and efficacy of TAC combined with low-dose corticosteroids in reducing proteinuria and preserving renal function in Chinese patients with Lee class III, IV and V IgAN.

\section{Materials and methods}

Patient selection. This was a retrospective study performed in Chinese patients with primary refractory $\operatorname{IgAN}$. The study was approved by the Ethics Committee of Shenzhen Second People's Hospital (Shenzhen, China) and all participating patients gave written, informed consent. All experiments conformed to the guidelines outlined by the 1964 Declaration of Helsinki.

Included subjects were 18-65 years old when a diagnosis of IgAN was confirmed by renal biopsy in the previous 3 months at Shenzhen Second People's Hospital. All patients were without a diagnosis of hepatic, cardiac, pulmonary or intestinal disease; had a glomerular filtration rate (GFR) within $30-60 \mathrm{ml} / \mathrm{min} / 1.73 \mathrm{~m}^{2}$, estimated by the Cockroft-Gault formula (20); proteinuria >1 g/day; and hypertension defined as systolic blood pressure $>140 \mathrm{mmHg}$ or diastolic blood pressure $>90 \mathrm{mmHg}$ associated with proteinuria between 0.3-1 g/day. All cases were reported as Lee class III, IV or V with renal dysfunction according to Lee's classification and histological assessment $(21,22)$. Exclusion criteria were as follows: i) Patients who were confirmed to have human immunodeficiency virus or hepatitis $\mathrm{B} / \mathrm{C}$ virus infection; ii) treatment with any other immunosuppressant in the previous 2 years; iii) use of other medication under research; iv) evidence or suspicion of renovascular disease, diabetes mellitus or poor control of hypertension; v) GFR $<30 \mathrm{ml} / \mathrm{min} / 1.73 \mathrm{~m}^{2}$ and serum bilirubin $>2 \mathrm{mg} / \mathrm{dl}$.

Treatment protocol. All patients with IgAN (male/female, 9:19; mean age, $48.32 \pm 10.06$ years) who were registered for a follow-up examination at Shenzhen Second People's Hospital received intravenous TAC (Astellas Pharma, Inc., Shenyang, China) combined with oral low-dose corticosteroids (GlaxoSmithKline Co., Ltd., Brentford, UK). After obtaining informed consent from each subject, all patients were treated with TAC and corticosteroid. In total, 28 patients were included in the study. Among them, 26 cases received TAC and prednisone, and the remaining 2 cases received TAC and methylprednisolone. In addition, all patients received the angiotensin inhibition medication, in the form of oral enalapril (2.5 mg once daily; Hisun Pharmaceutical Co., Ltd., Zhejiang, China) . TAC was started at a dose of $0.05 \mathrm{mg} / \mathrm{kg} / \mathrm{day}$, titrated to achieve a trough blood level of $5-10 \mathrm{ng} / \mathrm{ml}$ for the first 6 months, and then reduced to $4-6 \mathrm{ng} / \mathrm{ml}$ for the subsequent 6 months. Corticosteroids were started at a dose of $0.5 \mathrm{mg} / \mathrm{kg} / \mathrm{day}$ for the first 2 months, and then slowly tapered by $5 \mathrm{mg}$ every 2 weeks until discontinuation. Patients' records were reviewed from the start of the treatment through the time when the chart was reviewed and until the drug was discontinued or severe infection appeared (final time point).

Data collection and evaluation. Baseline characteristics including 24-h total urinary protein excretion, serum albumin, complete remission (CR), partial remission (PR), cholesterol, low-density lipoprotein (LDL), serum creatinine (Scr), blood glucose level and estimated GFR (eGFR) according to the modified Modification of Diet in Renal Disease (MDRD) equation for Chinese patients (23) were recorded and measured using a fully automated biochemical analyzer (Mindray, Shenzen, China). These data were collected at 3, 6 and 12 months after the initiation of treatment. The primary endpoints were complete remission (CR) and partial remission (PR). CR was defined as the disappearance of all known targeted initial spots, and normal laboratory and imaging parameters. PR was defined as a decrease of $\geq 30 \%$ (but not $100 \%$ ) of the sum of the longest diameters of the predetermined target lesions with no novel or enlarging lesions. Secondary endpoints included change of Scr, eGFR, clinical data and adverse events.

Histological diagnosis. Histological diagnosis of $\operatorname{Ig} \mathrm{AN}$ was based upon the demonstration of mesangioproliferative changes on light microscopy and the concomitant presence of predominant or codominant mesangial deposition of IgA. The pathological diagnosis was made by pathologists from Guangzhou KingMed Centre for Clinical Laboratory Co., Ltd. in accordance with the classification described by Lee et al (21).

Statistical analysis. The response rate, total urinary protein excretion, serum albumin, LDL, cholesterol, Scr and eGFR results were compared at baseline and every follow-up time point (3, 6 and 12 months) in all patients. The total urinary protein excretion, serum albumin, LDL, cholesterol, Scr, blood glucose level and eGFR are presented as the mean \pm standard deviation, and differences from baseline were evaluated using a Student's t-test. The response rate is presented as the percentage of patients exhibiting $\mathrm{CR}$ or PR. The response rate results for total urinary protein excretion were compared between baseline and each follow-up time using the $\chi^{2}$-test. In all analyses, SPSS version 13.0 software (SPSS, Inc., Chicago, IL, USA) was used. $\mathrm{P}<0.05$ was considered to indicate a statistically significant difference.

\section{Results}

Changes in clinical findings over time. Changes in total urinary protein excretion, serum albumin, cholesterol, LDL, response rate and blood glucose level were investigated at various times after the initiation of treatment. At different time points of follow-up, the total urinary protein excretion, response rate of proteinuria, and serum albumin, cholesterol and LDL levels were each improved, (Table I). After 12 months, CR was achieved in $40.1 \%$ of patients and PR in $43.4 \%$, yielding a total response rate of $83.5 \%$. Total urinary protein excretion $(0.40 \pm 0.38$ vs. $1.94 \pm 1.45 \mathrm{~g} / \mathrm{day} ; \mathrm{P}=0.002)$, 
Table I. Clinical and laboratory data at different follow-up time points.

\begin{tabular}{lcrrr}
\hline Clinical and laboratory data & Baseline & 3 months & 6 months & 12 months \\
\hline UTP (g/day) & $1.94 \pm 1.45$ & $0.64 \pm 0.36$ & $0.56 \pm 0.38$ & $0.40 \pm 0.38$ \\
Serum albumin (g/l) & $33.28 \pm 3.75$ & $38.89 \pm 4.31$ & $39.85 \pm 2.76$ & $39.82 \pm 3.68$ \\
Cholesterol (mmol/l) & $5.88 \pm 3.21$ & $5.03 \pm 2.26$ & $4.35 \pm 3.16$ & $4.68 \pm 2.23$ \\
LDL (mmol/l) & $2.35 \pm 1.23$ & $2.33 \pm 1.46$ & $2.01 \pm 1.16$ & $1.83 \pm 1.02$ \\
Response rate (CR; PR) (\%) & - & $71.4(26.4 ; 45)$ & $82.7(30.9 ; 51.8)$ & $83.5(40.1 ; 43.4)$ \\
Blood glucose level (mmol/l) & $5.11 \pm 2.23$ & $5.08 \pm 1.96$ & $5.28 \pm 1.83$ & $5.33 \pm 2.16$ \\
\hline
\end{tabular}

UTP, urine total protein; LDL, low-density lipoprotein; CR, complete remission; PR, partial remission.

Table II. Serum creatinine and eGFR levels at different follow-up time points (mean \pm standard deviation).

\begin{tabular}{|c|c|c|c|c|}
\hline Renal function data & Baseline & 3 months & 6 months & 12 months \\
\hline $\operatorname{Scr}(\mu \mathrm{mol} / 1)$ & $136.57 \pm 73.71$ & $133.37 \pm 81.12^{\mathrm{a}}$ & $116.91 \pm 36.98^{b}$ & $106.33 \pm 53.88^{c}$ \\
\hline eGFR $\left(\mathrm{ml} / \mathrm{min} / 1.73 \mathrm{~m}^{2}\right)$ & $71.32 \pm 44.71$ & $71.89 \pm 38.57^{\mathrm{d}}$ & $75.18 \pm 41.86^{\mathrm{e}}$ & $80.17 \pm 32.33^{f}$ \\
\hline
\end{tabular}

eGFR, estimated glomerular filtration rate; Scr, serum creatinine. ${ }^{\mathrm{a}} \mathrm{P}=0.257,{ }^{\mathrm{b}} \mathrm{P}=0.039,{ }^{\mathrm{c}} \mathrm{P}=0.023 ;{ }^{\mathrm{d}} \mathrm{P}=0.727,{ }^{\mathrm{e}} \mathrm{P}=0.396,{ }^{\mathrm{f}} \mathrm{P}=0.027$.

serum albumin $(39.82 \pm 3.68$ vs. $33.28 \pm 3.75 \mathrm{~g} / 1 ; \mathrm{P}=0.023)$, cholesterol $(4.68 \pm 2.23$ vs. $5.88 \pm 3.21 \mathrm{mmol} / 1 ; \mathrm{P}=0.043)$ and LDL $(1.83 \pm 1.02$ vs. $2.35 \pm 1.23 \mathrm{mmol} / \mathrm{l} ; \mathrm{P}=0.041)$ were improved significantly compared with the respective baseline values. Proteinuria (urinary protein excretion, $0.64 \pm 0.36$ vs $1.94 \pm 1.45 \mathrm{~g} /$ day; $\mathrm{P}=0.024)$ and serum albumin $(38.89 \pm 4.31 \mathrm{vs}$. $33.28 \pm 3.75 \mathrm{~g} / \mathrm{l} ; \mathrm{P}=0.043$ ) were already significantly improved by month 3 of treatment. The blood glucose level was stable over time.

Preservation of renal function. At month 12 of follow-up, the renal function remained stable or was improved compared with the baseline level as evidenced by serum eGFR $\left(80.17 \pm 32.33\right.$ vs. $\left.71.32 \pm 44.71 \mathrm{ml} / \mathrm{min} / 1.73 \mathrm{~m}^{2} ; \mathrm{P}=0.027\right)$ and Scr $(106.33 \pm 53.88$ vs. $136.57 \pm 73.71 \mu \mathrm{mol} / 1 ; \mathrm{P}=0.023)$ (Table II). The serum Scr levels decreased as the time after the start of treatment increased, with a significant difference from the baseline value at each time point, while the eGFR increased significantly over time in comparison with the baseline levels value (all $\mathrm{P}<0.05$ ).

Relapse following the discontinuation of TAC and corticosteroids. Two cases $(7.1 \%)$ of relapse were observed after cessation of the regimen within 6 months. The time to relapse ranged from 3 to 6 months.

Adverse events. One case of pneumococcal pneumonia developed in a patient treated with TAC combined with low-dose methylprednisolone at $\sim 6$ months after the initiation of this regimen, and one case of upper gastrointestinal hemorrhage (duodenal ulcer) was found in a patient treated with TAC plus low-dose prednisone at $\sim 4$ weeks after the initiation of treatment. The two patients with side effects were completely recovered following timely treatment of TAC combined with low-dose methylprednisolone.

\section{Discussion}

Proteinuria has been shown to be an adverse prognostic factor in renal diseases, including $\operatorname{Ig} \mathrm{AN}$; previous studies have established the importance of achieving a PR of proteinuria in patients with $\operatorname{IgAN}(24,25)$. Blockade of the renin-angiotensin system (RAS) can be considered the most important strategy for the treatment of proteinuria in patients with $\operatorname{IgAN}(26)$. In patients with proteinuria $>1 \mathrm{~g}$ /day following a full dose of an RAS blocking agent, steroids and/or immunosuppressive agents are suggested (27). However, some patients continue to present with massive proteinuria following therapy with steroids and/or immunosuppressive agents combined with RAS blockade (28). The identification of an effective therapeutic strategy for IgAN patients with refractory proteinuria is extremely important.

TAC (also known as FK506 or fujimycin) inhibits the activation of a transcription factor essential for the transcription of cytokine genes in $\mathrm{T}$ cells, causing the production of cytokines such as IL- 2 and IFN- $\gamma$ to be reduced, and ultimately resulting in immune suppression (29). Since the activation of T cells plays a crucial role in the pathogenesis of inflammatory glomerular diseases (30). TAC has been used as a therapeutic agent in various glomerular diseases, particularly refractory nephrotic syndrome. In a clinical trial, the efficacy and safety of a combination of TAC and a moderate dose of steroids were observed in a group of 14 patients who were confirmed as having refractory IgAN (19). However, the safety and efficacy of TAC combined with low-dose corticosteroids in patients with refractory IgAN has not been fully evaluated.

The present study, with a long-term follow-up, showed that the combination of TAC and a low-dose of corticosteroid contributed to a CR of proteinuria in $40.1 \%$ of patients and PR in $43.4 \%$, yielding a total response rate of $83.5 \%$. One possible explanation is that this immunosuppressive regimen significantly limited glomerular epithelial cell proliferation and inhibited crescent 
formation in nephropathy (31). Urine total protein excretion, serum albumin, cholesterol and LDL results were improved significantly compared with those at baseline. Proteinuria and serum albumin were significantly improved by month 3 of treatment. Two patients relapsed during the subsequent 3 months of follow-up. At the 12-month follow-up time point, renal function was totally stable compared with the baseline level as evidenced by eGFR and serum creatinine results. The blood glucose level was stable. Roberti et al (32) and Li et al (33) reported that TAC was able to reduce proteinuria rapidly in adults and children with steroid-resistant nephritic syndrome, and the results of the present study are consistent with this.

Until now, the safety of TAC in the treatment of patients with $\operatorname{Ig} \mathrm{AN}$ and chronic renal function impairment was not well known. The main side effects of TAC reported previously include gastrointestinal symptoms such as abdominal pain and nausea, hypertension and increased Scr (19). In the present study, one case of pneumococcal pneumonia developed in a patient treated with TAC combined with low-dose methylprednisolone at $\sim 6$ months after the initiation of this regimen and one case of upper gastrointestinal hemorrhage (duodenal ulcer) was found in a patient treated with TAC plus low-dose prednisone at $\sim 4$ weeks after the initiation of treatment. The two patients with side effects were completely recovered after receiving appropriate treatment. TAC reduces insulin secretion by damaging the ultra-structure of beta cells in a time- or dose-dependent manner. It also decreases insulin mRNA levels by the reversible inhibition of calcineurin after binding to FK506-binding protein 12 (34). With respect to this, TAC theoretically has the potential to induce transient diabetes mellitus via defective insulin secretion (35), even resulting in diabetic ketoacidosis (36). However, in the present study the blood glucose level was stable at the 12-month follow-up. In this study group, TAC combined with low-dose corticosteroids exhibited very few toxic side effects, and showed promising preliminary efficacy with a high safety profile in Chinese patients with IgAN. However, the adverse effects cannot be ignored, and close monitoring of blood drug concentration and blood glucose levels, as well as regular evaluation of renal function are all required in future courses of treatment.

This retrospective study showed that TAC combined with low-dose corticosteroids was able to reduce proteinuria and preserve renal function in Chinese patients with Lee class III, IV or V IgAN. However, given the limitation of the small number of patients in this trial, a further study with a larger sample of participants and longer follow-up of period is required to provide more conclusive results.

\section{Acknowledgements}

The authors would like to acknowledge the reviewers for their helpful comments on this paper.

\section{References}

1. Karnib HH, Gharavi AG, Aftimos G, Mahfoud Z, Saad R, Gemayel E, Masri B, Assaad S, Badr KF and Ziyadeh FN: A 5-year survey of biopsy proven kidney diseases in Lebanon: Significant variation in prevalence of primary glomerular diseases by age, population structure and consanguinity. Nephrol Dial Transplant 25: 3962-3969, 2010.
2. Goto M, Wakai K, Kawamura T, Ando M, Endoh M and Tomino Y: A scoring system to predict renal outcome in $\operatorname{IgA}$ nephropathy: A nationwide 10-year prospective cohort study. Nephrol Dial Transplant 24: 3068-3074, 2009.

3. Kiryluk K, Li Y, Sanna-Cherchi S, Rohanizadegan M, Suzuki H, Eitner F, Snyder HJ, Choi M, Hou P, Scolari F, et al: Geographic differences in genetic susceptibility to IgA nephropathy: GWAS replication study and geospatial risk analysis. PLoS Genet 8: e1002765, 2012.

4. Chacko B: IgA nephropathy in India: What we do know. Ren Fail 33: 102-107, 2011.

5. Yamamoto R, Nagasawa $Y$, Shoji $T$, Inoue $K$, Uehata $T$, Kaneko T, Okada T, Yamauchi A, Tsubakihara Y, Imai E, et al: A candidate gene approach to genetic prognostic factors of $\operatorname{IgA}$ nephropathy-a result of polymorphism research to distinguish genetic factors contributing to progression of $\operatorname{IgA}$ nephropathy (PREDICT-IgAN). Nephrol Dial Transplant 24: 3686-3694, 2009.

6. Kaartinen K, Syrjänen J, Porsti I, Harmoinen A, Pasternack A, Huhtala $\mathrm{H}$, Niemelä $\mathrm{O}$ and Mustonen J: Insulin resistance and the progression of IgA glomerulonephritis. Nephrol Dial Transplant 22: 778-783, 2007.

7. Bonnet F, Deprele C, Sassolas A, Moulin P, Alamartine E, Berthezène $F$ and Berthoux F: Excessive body weight as a new independent risk factor for clinical and pathological progression in primary IgA nephritis. Am J Kidney Dis 37: 720-727, 2001.

8. Chen W, Liu Q, Liao Y, Yang Z, Chen J, Fu J, Zhang J, Kong Y, $\mathrm{Fu}$ P, Lou T, et al: Outcomes of tacrolimus therapy in adults with refractory membranous nephrotic syndrome: A prospective, multicenter clinical trial. Am J Med Sci 345: 81-87, 2013.

9. Loeffler K, Gowrishankar M and Yiu V: Tacrolimus therapy in pediatric patients with treatment-resistant nephrotic syndrome. Pediatr Nephrol 19: 281-287, 2004.

10. Girman P, Lipar K, Koznarova R, Boucek P, Kriz J, Kocik M, Havrdova T, Adamec M and Saudek F: Similar early complication rate in simultaneous pancreas and kidney recipients on tacrolimus/mycophenolate mofetil versus tacrolimus/sirolimus immunosuppressive regimens. Transplant Proc 42: 1999-2002, 2010.

11. Naesens M, Kuypers DR and Sarwal M: Calcineurin inhibitor nephrotoxicity. Clin J Am Soc Nephrol 4: 481-508, 2009.

12. Flechner SM, Kobashigawa J and Klintmalm G: Calcineurin inhibitor-sparing regimens in solid organ transplantation: Focus on improving renal function and nephrotoxicity. Clin Transplant 22: 1-15, 2008.

13. Wierzbicka A, Pawłowska J, Socha P, Jankowska I, Skorupa E, Teisseyre M, Ismail H, Czubkowski P and Socha J: Lipid, carbohydrate metabolism and antioxidant status in children after liver transplantation. Transplant Proc 39: 1523-1525, 2007.

14. Noto T, Furuichi Y, Ishiye M, Matsuoka N, Aramori I, Mutoh S and Yanagihara T: Tacrolimus (FK506) limits accumulation of granulocytes and platelets and protects against brain damage after transient focal cerebral ischemia in rat. Biol Pharm Bull 30: 313-317, 2007.

15. Aomatsu T, Imaeda H, Takahashi K, Fujimoto T, Kasumi E, Yoden A, Tamai H, Fujiyama Y and Andoh A: Tacrolimus (FK506) suppresses TNF- $\alpha$-induced CCL2 (MCP-1) and CXCL10 (IP-10) expression via the inhibition of p38 MAP kinase activation in human colonic myofibroblasts. Int J Mol Med 30: 1152-1158, 2012.

16. Faul C, Donnelly M, Merscher-Gomez S, Chang YH, Franz S, Delfgaauw J, Chang JM, Choi HY, Campbell KN, Kim K, et al: The actin cytoskeleton of kidney podocytes is a direct target of the antiproteinuric effect of cyclosporine A. Nat Med 14: 931-938, 2008.

17. Mathieson PW: Proteinuria and immunity-an overstated relationship? N Engl J Med 359: 2492-2494, 2008.

18. Rostaing L, Cantarovich D, Mourad G, Budde K, Rigotti P, Mariat C, Margreiter R, Capdevilla L, Lang P, Vialtel P, et al: Corticosteroid-free immunosuppression with tacrolimus, mycophenolate mofetil and daclizumab induction in renal transplantation. Transplantation 79: 807-814, 2005.

19. Zhang Q, Shi SF, Zhu L, Lv JC, Liu LJ, Chen YQ, Zhang H and Wang HY: Tacrolimus improves the proteinuria remission in patients with refractory $\operatorname{IgA}$ nephropathy. Am J Nephrol 35: 312-320, 2012.

20. Bayrak O, Seckiner I, Erturhan SM, Mizrak S and Erbagci A: Analysis of changes in the glomerular filtration rate as measured by the Cockroft-Gault formula in the early period after percutaneous nephrolithotomy. Korean J Urol 53: 552-555, 2012. 
21. Lee HS, Lee MS, Lee SM, Lee SY, Lee ES, Lee EY, Park SY, Han JS, Kim S and Lee JS: Histological grading of IgA nephropathy predicting renal outcome: Revisiting $\mathrm{H}$. S. Lee's glomerular grading system. Nephrol Dial Transplant 20: 342-348, 2005.

22. Lee SM, Rao VM, Franklin WA, Schiffer MS, Aronson AJ, Spargo BH and Katz AI: IgA nephropathy: Morphologic predictors of progressive renal disease. Hum Pathol 13: 314-322, 1982.

23. Ma YC, Zuo L, Chen JH, Luo Q, Yu XQ, Li Y, Xu JS, Huang SM, Wang LN, Huang W, et al: Modified glomerular filtration rate estimating equation for Chinese patients with chronic kidney disease. J Am Soc Nephrol 17: 2937-2944, 2006.

24. Ferraro PM, Ferraccioli GF, Gambaro G, Fulignati P and Costanzi S: Combined treatment with renin-angiotensin system blockers and polyunsaturated fatty acids in proteinuric $\operatorname{IgA}$ nephropathy: A randomized controlled trial. Nephrol Dial Transplant 24: 156-160, 2009.

25. Reich HN, Troyanov S, Scholey JW and Cattran DC; Toronto Glomerulonephritis Registry: Remission of proteinuria improves prognosis in IgA nephropathy. J Am Soc Nephrol 18: 3177-3183, 2007.

26. Manno C, Torres DD, Rossini M, Pesce F and Schena FP: Randomized controlled clinical trial of corticosteroids plus ACE-inhibitors with long-term follow-up in proteinuric IgA nephropathy. Nephrol Dial Transplant 24: 3694-3701, 2009.

27. Lv J, Zhang H, Chen Y, Li G, Jiang L, Singh AK and Wang H: Combination therapy of prednisone and ACE inhibitor versus ACE-inhibitor therapy alone in patients with IgA nephropathy: A randomized controlled trial. Am J Kidney Dis 53: 26-32, 2009.

28. Aliabadi AZ, Pohanka E, Seebacher G,Dunkler D, Kammerstätter D, Wolner E, Grimm M and Zuckermann AO Development of proteinuria after switch to sirolimus-based immunosuppression in long-term cardiac transplant patients. Am J Transplant 8: 854-861, 2008.
29. Skytte DM, Jaroszewski JW, Johansen KT, Hansen SH, Hansen L, Nielsen PG and Frydenvang K: Some transformations of tacrolimus, an immunosuppressive drug. Eur J Pharm Sci 48: 514-522, 2013.

30. Westhoff TH and van der Giet M: Tacrolimus in the treatment of idiopathic nephrotic syndrome. Expert Opin Investig Drugs 16: 1099-1110, 2007.

31. Chen M, Li H, Li XY, Lu FM, Ni ZH, Xu FF, Li XW, Chen JH and Wang HY; Chinese Nephropathy Membranous Study Group: Tacrolimus combined with corticosteroids in treatment of nephrotic idiopathic membranous nephropathy: A multicenter randomized controlled trial. Am J Med Sci 339: 233-238, 2010

32. Roberti I and Vyas S: Long-term outcome of children with steroid-resistant nephrotic syndrome treated with tacrolimus. Pediatr Nephrol 25: 1117-1124, 2010.

33. Li X, Li H, Ye H, Li Q, He X, Zhang X, Chen Y, Han F, $\mathrm{He} \mathrm{Q}$, Wang $\mathrm{H}$ and Chen J. Tacrolimus therapy in adults with steroid- and cyclophosphamide-resistant nephrotic syndrome and normal or mildly reduced GFR. Am J Kidney Dis 54: 51-58, 2009.

34. van Hooff JP, Christiaans MH and van Duijnhoven EM: Tacrolimus and posttransplant diabetes mellitus in renal transplantation. Transplantation 79: 1465-1469, 2005.

35. Dittrich K, Knerr I, Rascher W and Dötsch J: Transient insulin-dependent diabetes mellitus in children with steroid-dependent idiopathic nephrotic syndrome during tacrolimus treatment. Pediatr Nephrol 21: 958-961, 2006.

36. Sarkar S, Mondal R, Nandi M and Das AK: Tacrolimus induced diabetic ketoacidosis in nephrotic syndrome. Indian J Pediatr 80: 596-597, 2013. 